\title{
Thermal imaging observations of the surface of a gauge block
}

\author{
Krzysztof Dziarski ${ }^{1, *}$ \\ ${ }^{1}$ Institute of Metrology and Optoelectronics, Poznan University of Technology, ul. Piotrowo 3A 60-965 Poznań, Poland
}

\begin{abstract}
This paper proposes a method for determining the time at which the temperature of surface of a gauge block will be established. The relationship between the temperature of a gauge block, its length and the value of uncertainty of measurement when calibrating using this block was explained. Attention was paid to problems resulting from the applied measurement method. Particular attention was paid to thermal imaging observations of the lateral surface of a gauge block
\end{abstract}

\section{Preface}

The gauge blocks are rectangular length standards. Most often they are made of tool steel. The dimensions of a gauge block are subject to change as a result of the change in the value of its temperature. A change in the temperature of a gauge block may be caused, among others, by a change in ambient temperature or by a touch of hand. The actual length of the gauge block can be expressed by equation (1).

$$
l_{x}=l_{s}+\delta l_{D}+\delta l_{c}+\delta l-L\left(\alpha^{\prime} x \delta_{t}+\delta_{a} x \Delta t^{\prime}\right)-\delta l_{v}
$$

where $l_{s}$ - the length of gauge block at the reference temperature $t_{0}=20^{\circ} \mathrm{C}$ given in the calibration certificate, $\delta l_{D}$ - change in the length of gauge block since the last calibration due to drift, $\delta l$ - measured difference in the length of both gauge blocks, $\delta l_{C}$ correction for non-linearity and inaccuracy of the comparator setting, $L$ - nominal length of gauge block, $\alpha$ '- mean value of the thermal expansion coefficient of both gauge blocks, $\delta l_{t}$ - temperature difference of gauge blocks, $\delta_{\alpha}$ - difference of the thermal expansion coefficients of both gauge blocks, $\Delta \mathrm{t}^{\prime}$ - deviation of the mean temperature of gauge block and a plate calibrated from the ambient temperature, $\delta l_{v}$ - correction for the non-centricity of contact with the measuring surfaces of gauge block.

While analysing the above equation one can notice the effect of temperature of gauge blocks on the determined difference in length between the gauge block and calibrated plate and the uncertainty of the difference measured. For this reason, in order to measure the difference between the length of gauge block and the calibrated plate, the temperature of calibrated plate should be equalized with the temperature of gauge block. The aim of the research conducted was to determine the time in which the temperature of lateral surface of gauge block is established.2

\section{Methodology of conducted works}

In the works conducted, essential is the selection of method by which the temperature of lateral surface of the gauge block was recorded. In order to obtain a reliable distribution of temperature on the lateral surface of gauge blocked, a large number of measuring sensors should be placed. This would increase the thermal capacity of the entire system (calibrated plate and measuring sensors). Consequently, the time in which the temperature of plate with sensors placed would have reached the ambient temperature would differ from the time in which the plate without the sensors would have reached the ambient temperature. Impossibility to obtain appropriate thermal connections between the surface of gauge block and the surface of sensor should also be mentioned. In order to protect the plate from damage caused by the effect of chemicals on the structure of plate, it was impossible to use thermally conductive paste. Due to the above difficulties, it was decided to use thermal imaging. This non-contact method allows eliminating the problems described above. However, it is not free of drawbacks. The uncertainty measurement of the thermal imaging camera is greater than the uncertainty of measurement made using the contact method. The temperature distribution on the surface of gauge block was examined using a Flir E50 thermal imaging camera fitted with micro bolometric infrared sensors [2].

\section{Determining the time to establish the temperature of surface of plate}

To ensure a stable ambient temperature, the gauge block was placed inside the ILW115 climate chamber. The temperature of interior of the chamber corresponded to the temperature of room in which the gauge blocks are stored and where the plate is compared with gauge blocks. In the first part of the experiment, it was required 
to determine the time in which the temperature of plate will equal to the temperature inside the chamber.

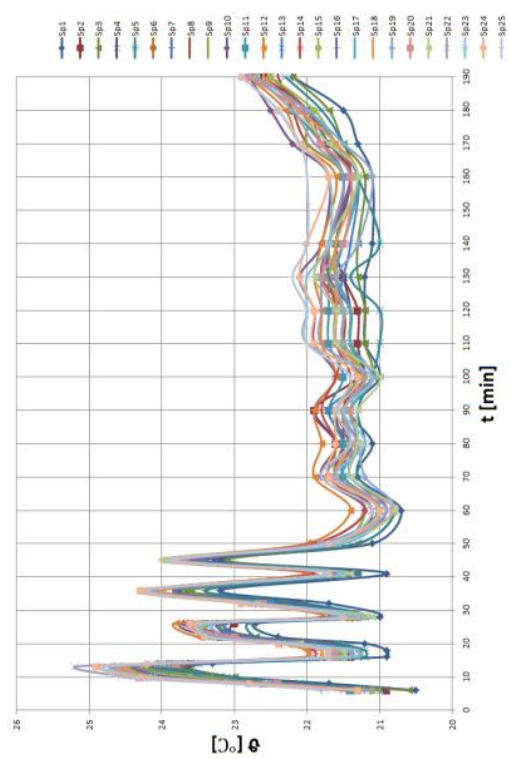

Fig.1 . Temperature of the lateral surface of gauge block during the experiment. In time $t=0$, the gauge block was placed inside a climate chamber.

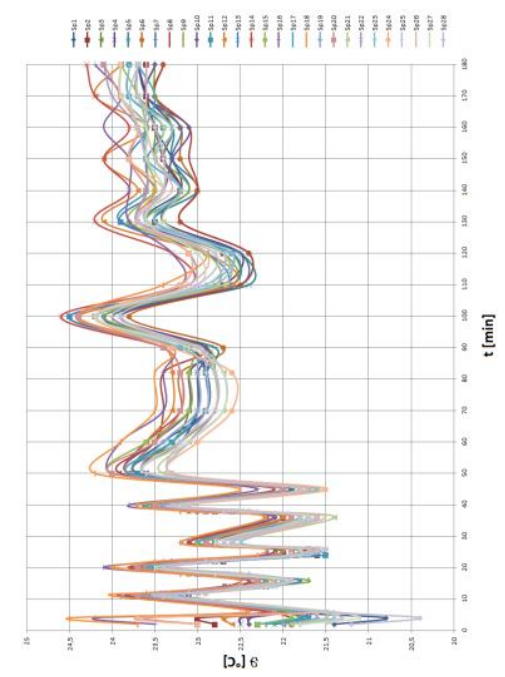

Fig.2 . Temperature of the lateral surface of gauge block after being touched by a hand. In time $t=0$, the plate (czy płytki wzorcowej - wówczas gauge block) was placed in a climate chamber.

For this purpose, the plate was placed vertically inside the climate chamber. The interior of chamber was lined with black cardboard. In addition, the plate was placed in such a way, that it was aside the recorded thermogram and was reversed at an angle of $30^{\circ} \mathrm{C}$ [1] in relation to the lens of camera.. This allowed to avoid distorting the recorded temperature distribution on the surface of gauge block resulting from the effect of reflected radiation. The distribution of temperature on the surface of reference block was recorded at selected points, evenly distributed over the plate surface, for approximately three hours. The temperature value measured at the designated points was read out every 10 minutes. The results obtained are shown in Fig. 1 In the second part of the work performed, the time was determined which elapsed from touching the gauge block with hand till the temperature of gauge block equalled the temperature inside the chamber. For this purpose, the gauge block was placed in a climate chamber. After elapsing the time of 40 minutes, the gauge block was gripped by the hand and held for 1 minute. Then the surface of gauge block was observed again for 180 minutes. The results obtained are shown in Figure 2.

\section{Summary}

The research carried out confirmed that the temperature of gauge block is established within the entire volume after three hours. It was also found that the temperature of gauge block changed as a result of touching with hand for 1 minute would also be established within three hours. During the work carried out, it was noticed that the use of climate chamber did not allow to fully reflect the conditions in the room where the plates were compared. Due to the limited volume of chamber and the mixing of air masses with different temperatures inside the chamber, it is not possible to reach the same temperature values by the entire plate. The fact of reaching by the plate the established temperature was proven by the lowered amplitude of temperature fluctuations of each point of plate below the value set by the highest and lowest value of chamber temperature recorded in the course of work. It should be remembered that the measurement made with the use of a thermal imaging camera, like the measurement made with the use of another device, is fraught with uncertainty $[3,4]$. The presented works were long-lasting. For this reason, the presented measurement system can be expanded and controlled from the PC level [5], while the measurement results can be sent via wireless methods [6].

\section{References}

1. K. Dziarski, J. Parzych, The solutions used for long wave thermographic cameras designed for observing elements in SMD housings, J. ITM WOC Vol. 19, 2018

2. M. Litwa, G. Wiczyński, Effect of the observation angle on the result of temperature measurement by thermal imaging camera, Elektronika 2008, Vol. 49, no.6, pp. 147-148

3. A. Odon, Z. Krawiecki, Meas., 44, 8, 1406-1411, (2011)

4. A. Hulewicz, Prz. Elektrotechniczny, 11a, 319 (2010)

5. P. Otomanski, Z. Krawiecki, A. Odon, JPCS, 238, 012005 (2010).

6. A. Cysewska-Sobusiak, A. Hulewicz, M. Boltrukiewicz, D. Prokop, Proc. IEEE VECIMS, $116(205)$ 